\title{
Variação temporal dos atributos microbiológicos do solo sob diferentes usos
}

\section{Time variation of soil microbiological attributes under different uses}

\author{
Elaine Novak*, Laércio A. Carvalho, Etenaldo F. Santiago, Amanda V. Brumatti, \\ Leonardo L. Santos e Larissa C. Sales
}

Universidade Estadual de Mato Grosso do Sul - Cidade Universitária de Dourados, MS, Brasil - Caixa Postal 351 - CEP: 79804-970, Brasil

(`E-mail:elainenovak_@hotmail.com)

http://dx.doi.org/10.19084/RCA17300

Recebido/received: 2017.11.22

Recebido em versão revista/received in revised form: 2018.02.05

Aceite/accepted: 2018.02.28

\begin{abstract}
R E S U M O
Compreender o comportamento e a dinâmica dos microrganismos do solo ajuda a avaliar as práticas de uso do solo que mantém e/ou melhoram a qualidade deste ao longo do tempo. O objetivo deste trabalho foi avaliar a qualidade do solo de diferentes áreas com base na biomassa microbiana, respiração basal e índices derivados (quociente metabólico e quociente microbiano) em dois períodos de amostragem. Quatro amostras compostas oriundas de três subamostras de solo foram coletadas nos anos de 2012 e 2014 na camada de 0,00-0,10 m em sete áreas distintas, sendo cinco áreas em restauração ecológica, um remanescente de floresta nativa e uma área de cultivo de cana-de-açúcar. Os dados foram submetidos à análise de variância, aplicando-se fatorial, sendo as médias comparadas pelo teste de Tukey, a 5\% de significância e análise multivariada, por meio da agrupamento (Cluster Analysis). Os resultados indicam que, embora o tempo de resiliência das áreas em restauração ecológica seja relativamente curto, o desenvolvimento da cobertura vegetal e o estabelecimento da comunidade microbiana foram importantes para promover a melhoria da qualidade do solo.
\end{abstract}

Palavras-chave: Atividade Microbiana, Monitorização do solo, Restauração ecológica, Cultivo de cana-de-açúcar.

\begin{abstract}
A B S T R A C T
Understanding the behavior and dynamics of soil microorganisms helps to evaluate land use practices that maintain and / or improve soil quality over time. The objective of this study was to evaluate soil quality in different areas based on microbial biomass, basal respiration and derivatives indices (metabolic quotient and microbial quotient) in two sampling periods. Four composed samples from three soil subsamples were collected in the 2012 and 2014 period in the layer of 0.00 to $0.10 \mathrm{~m}$ in seven distinct areas, with five areas in ecological restoration, a native forest remnant and an area of sugarcane cultivation. Data were subjected to analysis of variance, applying factorial, and the averages compared by Tukey test at $5 \%$ significance and multivariate by cluster analysis. The results indicate that, although the resilience of ecological restoration areas is relatively short, the development of the vegetation cover and the establishment of the microbial community were important to promote the improvement of soil quality.
\end{abstract}

Keywords: Microbial Activity, Soil monitoring, Ecological restoration, Sugarcane cultivation.

\section{INTRODUÇÃO}

Processos de degradação atuam sobre os diferentes compartimentos no ecossistema. Dependendo da intensidade e duração do impacto e da resiliência do ambiente, a restauração poderá ocorrer de maneira natural ou necessitará de intervenção antrópica. Quando a degradação leva a perturbações do solo por ações naturais a resiliência natural do ambiente geralmente possibilita a sua recuperação. No entanto, a degradação associada com perda da camada superficial do solo contendo matéria orgânica e nutrientes em maior quantidade é mais séria, considerando a importância destes para as características físicas, químicas e biológicas do solo (Dias et al., 2007). 
A restauração de áreas degradadas envolve uma série de medidas que buscam, inicialmente, minimizar os efeitos da degradação e, posteriormente, implantar metodologias corretivas, que visam à rápida recuperação do ambiente (Novak et al., 2017). Entretanto, antes da adoção das diferentes medidas mitigadoras, é de extrema importância a avaliação dos atributos do solo, pois, retratam o seu diagnóstico atual e verificam se os métodos empregados estão adequados às condições específicas da atividade (Audeh et al., 2011) e da região, tornando possível um melhor planeamento e execução de práticas de uso e ocupação.

Alguns atributos microbiológicos do solo são mais sensíveis a alterações quando o solo está sujeito às diferentes formas de manejo, podendo ser estes considerados bons indicadores de qualidade do solo (Souza et al., 2012). Entre os atributos microbiológicos, a biomassa microbiana do solo (C-BMS) é um importante parâmetro de ciclagem de nutrientes nos ecossistemas, pois representa a fração da matéria orgânica do solo (MOS) que é mais rapidamente decomposta (Rangel-Vasconcelos et al., 2015), permitindo a avaliação mais rápida e precoce de suas alterações (García-Orenes et al., 2013), quando comparada aos atributos químicos e físicos (Hungria et al., 2009; Kaschuk et al., 2010).

A respiração basal reflete a velocidade de decomposição de um resíduo orgânico adicionado ao solo. Quando essa atividade microbiana é alta ocorre maior decomposição e, consequentemente, liberação de nutrientes para as plantas, porém, também pode significar perdas de carbono do solo, a longo prazo (Souza et al., 2010).

No geral, informações sobre as mudanças no carbono da biomassa microbiana (C-BMS), respiração basal $\left(\mathrm{C}-\mathrm{CO}_{2}\right)$, quociente metabólico $\left(q \mathrm{CO}_{2}\right)$ e quociente microbiano ( $q \mathrm{MIC}$ ) podem explicar os processos ecológicos do ambiente. Compreender o comportamento dos microrganismos do solo ajuda a avaliar as práticas de uso que mantém e/ ou melhoram a qualidade deste ao longo do tempo, pois mudanças na atividade microbiana e na composição das comunidades microbianas podem influenciar diretamente em sua fertilidade, bem como no crescimento das plantas (Crecchio et al., 2007).
Neste contexto, o objetivo deste trabalho foi avaliar a qualidade do solo de diferentes áreas com base na biomassa microbiana, respiração basal e índices derivados (quociente metabólico e quociente microbiano) em dois períodos de amostragem.

\section{MATERIAL E MÉTODOS}

O trabalho foi conduzido no município de Rio Brilhante, Estado de Mato Grosso do Sul $\left(21^{\circ} 48^{\prime}\right.$ S, 443' W). O solo é classificado como Latossolo Vermelho Eutrófico típico, de textura argilosa (Embrapa, 2013). De acordo com o sistema de classificação Köppen (Cwa), o clima é caracterizado como mesotérmico húmido, com verões quentes e invernos secos. As áreas de estudo consistiram em um remanescente de vegetação nativa, com fisionomia florística de área de transição entre Cerrado e Mata Atlântica, que foi adotada como área testemunha e identificada como MATA, além de cinco áreas em restauração ecológica (identificadas como REC1 a REC5) e uma área de cultivo de cana-de-açúcar $(\mathrm{CN})$.

As áreas em restauração foram estabelecidas originalmente em formação de Floresta Estacional Semidecidual e substituídas por pastagem (Brachiaria brizantha), manejadas num período de dez anos e, posteriormente, por cana-de-açúcar num período de 2 anos. Após este período, as áreas foram destinadas à recuperação ambiental. Os fragmentos destinados à recuperação implantados no ano de 2010 foram identificados como REC1 a REC5, com adoção de regeneração natural e plantio de espécies nativas florestais, tais como: Myracrodruon urundeuva, Schinus terebinthifolia, Handroanthus avellanedae, Machaerium stipitatum e Dabergia miscolobium, entre outras, na área identificada como REC2 e regeneração natural nas demais áreas. Em nenhuma das áreas houve intervenção mecânica e aplicação de fertilizantes.

O plantio da cana-de-açúcar (RB86 - 7515) foi realizado com preparação convencional do solo utilizando duas gradagens (grades aradoras), uma subsolagem até à profundidade de $0,45 \mathrm{~m}$ e uma gradagem niveladora. Foi utilizado fertilizante mineral NPK (05-15-10) durante o plantio da cultura e nos cortes anuais a reposição foi feita utilizando fertilizante mineral NPK (30-05-25) 
na linha da cultura. Nos tratos culturais da cana soca foram realizadas operações de cultivo entre linhas da cultura, visando minimizar os efeitos da compactação do solo causada pelo intensivo tráfego de máquinas na colheita.

Para avaliação dos atributos microbiológicos do solo, as amostras foram coletadas na camada 0,00$0,10 \mathrm{~m}$, com quatro repetições aleatórias em cada área seguindo o delineamento de blocos inteiramente casualizados, num esquema fatorial $7 \times 2$, sendo sete áreas avaliadas em dois períodos de amostragem (2012 e 2014).

O carbono da biomassa microbiana do solo (C-BMS) foi avaliado pelo método da fumigação-extração estabelecido por Vance et al. (1987). A respiração basal do solo $\left(\mathrm{C}_{-} \mathrm{CO}_{2}\right)$ foi obtida pelo método de fumigação-incubação proposto por Jenkinson e Powlson (1976). Após a realização das análises de C-BMS e C- $\mathrm{CO}_{2}$ evoluído, foram determinados os quocientes metabólico $\left(\mathrm{qCO}_{2}\right)$, obtido a partir da relação $\mathrm{C}-\mathrm{CO}_{2} / \mathrm{C}-\mathrm{BMS}$, e microbiano (qMIC), pela equação CBMS/COT (Carbono Orgânico Total) x 100 (Anderson e Domsch, 1990).

Os resultados dos atributos do solo avaliados foram submetidos à análise de variância (ANOVA), aplicando-se fatorial, sendo as médias comparadas pelo teste de Tukey, a 5\% de significância. Além disso, os parâmetros referentes à biomassa microbiana e aos índices derivados foram submetidos à análise de agrupamento (Cluster Analysis), adotando-se o método do vizinho mais distante (Complete Linkage), a partir da distância euclidiana, para descrever a similaridade entre as áreas estudadas.

\section{RESULTADOS E DISCUSSÃO}

Os atributos biológicos do solo comportaram-se de modo distinto nas diferentes áreas e períodos de amostragem. Pela análise de variância verificou-se que a interação das áreas estudadas e período de amostragem foi significativa $(\mathrm{p}<0,01)$ para o atributo $q \mathrm{CO}_{2}$ Para os demais atributos microbiológicos avaliados não houve interação das áreas estudadas e período de amostragem (Quadro 1).

A biomassa microbiana é considerada a parte viva e mais ativa da matéria orgânica do solo (Roscoe et al., 2006; Singh et al., 2011) e geralmente compreende 2 a $4 \%$ do carbono orgânico total (Gama-Rodrigues, 1999), sendo que valores menores que estes indicam perdas de carbono do sistema. Neste estudo, os teores de carbono da biomassa microbiana do solo (C-BMS) variaram entre $537,93 \mu \mathrm{g} \mathrm{C} \mathrm{g}{ }^{-1}$

Quadro 1 - Médias dos teores de carbono da biomassa microbiana do solo (C-BMS), respiração basal (C- $\left.\mathrm{CO}_{2}\right)$, quociente metabólico $\left(q \mathrm{CO}_{2}\right)$ e quociente microbiano $(q \mathrm{MIC})$ de áreas em restauração ecológica, cultivo de cana-de-açúcar e vegetação nativa nas épocas de amostragem 2012 e 2014

\begin{tabular}{|c|c|c|c|c|c|c|c|c|}
\hline \multirow[t]{2}{*}{ Área } & \multicolumn{2}{|c|}{ 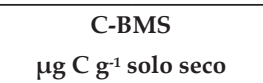 } & \multicolumn{2}{|c|}{$\begin{array}{c}\mathrm{C}-\mathrm{CO}_{2} \\
\mu \mathrm{g} \mathrm{C}-\mathrm{CO}_{2} \mathrm{~g}^{-1} \text { solo dia }-1\end{array}$} & \multicolumn{2}{|c|}{$\begin{array}{c}\mathrm{qCO}_{2} \\
\mu \mathrm{C}-\mathrm{O} 2 \mu \mathrm{g}^{-1} \mathrm{C}^{-B M S} \mathrm{~h}^{-1}\end{array}$} & \multicolumn{2}{|c|}{$\begin{array}{c}\text { qMIC } \\
\%\end{array}$} \\
\hline & 2012 & 2014 & 2012 & 2014 & 2012 & 2014 & 2012 & 2014 \\
\hline REC 1 & 248,81 & 406,45 & 24,58 & 31,67 & $42,27 \mathrm{bA}$ & $32,46 \mathrm{aA}$ & 0,70 & 1,36 \\
\hline REC 2 & 332,11 & 465,46 & 27,65 & 28,36 & $34,51 \mathrm{bA}$ & $25,92 \mathrm{aA}$ & 0,90 & 1,29 \\
\hline REC 3 & 405,16 & 389,25 & 27,70 & 26,14 & $27,74 \mathrm{bA}$ & $29,56 \mathrm{aA}$ & 1,17 & 1,09 \\
\hline REC 4 & 423,69 & 461,22 & 31,88 & 33,88 & $31,20 \mathrm{bA}$ & $30,88 \mathrm{aA}$ & 1,20 & 1,31 \\
\hline REC 5 & 305,79 & 386,42 & 29,49 & 23,13 & $41,26 \mathrm{bA}$ & $35,10 \mathrm{aA}$ & 1,25 & 1,32 \\
\hline $\mathrm{CN}$ & 100,76 & 309,34 & 24,64 & 29,07 & $141,91 \mathrm{aA}$ & $38,79 \mathrm{aB}$ & 0,32 & 1,26 \\
\hline MATA & 537,93 & 394,63 & 38,00 & 32,80 & $33,24 \mathrm{bA}$ & $38,05 \mathrm{aA}$ & 1,08 & 1,02 \\
\hline $\mathrm{F}_{\text {área }}$ & \multicolumn{2}{|c|}{$4.8031^{* *}$} & \multicolumn{2}{|c|}{$1.5167 \mathrm{~ns}$} & \multicolumn{2}{|c|}{$6.2168^{* *}$} & \multicolumn{2}{|c|}{$1.6297 \mathrm{~ns}$} \\
\hline $\mathrm{F}_{\text {ano }}$ & \multicolumn{2}{|c|}{$4.7397^{*}$} & \multicolumn{2}{|c|}{$0.0058 \mathrm{~ns}$} & \multicolumn{2}{|c|}{$6.8844^{*}$} & \multicolumn{2}{|c|}{$8.8972^{* *}$} \\
\hline$F_{\text {trat } x \text { ano }}$ & \multicolumn{2}{|c|}{$2.2302 \mathrm{~ns}$} & \multicolumn{2}{|c|}{$0.7421 \mathrm{~ns}$} & \multicolumn{2}{|c|}{$4.7794^{* *}$} & \multicolumn{2}{|c|}{$2.2294 \mathrm{~ns}$} \\
\hline C.V.(\%) & \multicolumn{2}{|c|}{30.50} & \multicolumn{2}{|c|}{27.53} & \multicolumn{2}{|c|}{59.38} & \multicolumn{2}{|c|}{33.44} \\
\hline
\end{tabular}

REC1 (área em restauração ecológica 1), REC2 (área em restauração ecológica 2), REC3 (área em restauração ecológica 3), REC4 (área em restauração ecológica 4),

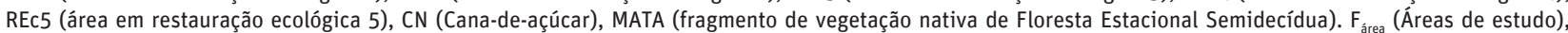
$\mathrm{F}_{\text {ano }}$ (Época de amostragem - 2012 e 2014), $\mathrm{F}_{\text {anea }}$ (Interação das áreas de estudo com as épocas de amostragem). ** significativo ao nível de $1 \%$ de probabilidade $(p<0,01)$, * significativo ao nível de $5 \%$ de probabilidade $(p<0,05)$ ns= não significativo. Médias seguidas com a mesma letra não diferem significativamente pelo teste de Tukey; letra minúscula compara médias nas colunas e letra maiúscula nas linhas. 


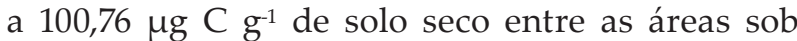
vegetação nativa e cultivo de cana-de-açúcar, respectivamente, no primeiro ano de amostragem (2012) e de 465,46 $\mu \mathrm{C} \mathrm{g}^{-1}$ a 309,34 $\mu \mathrm{g} \mathrm{C} \mathrm{g-1}$ de solo seco entre as áreas sob restauração ecológica (REC2) e cultivo de cana-de-açúcar $(\mathrm{CN})$, respectivamente, no segundo ano de amostragem (2014).

Com relação aos teores de C-BMS, a análise de variância indicou que há diferença significativa entre as épocas de amostragem $(\mathrm{p}<0,05)$, com maior média em 2014 (dado não apresentado). A substituição do sistema de cultivo de cana-de-açúcar por áreas em restauração ecológica resultou em aumento do C-BMS em 59,50\%, 69,66\%, 75,13\%, $76,21 \%, 67,04 \%$, na primeira época de amostragem, e de $23,89 \%, 33,32 \%, 20,53 \%, 32,93 \%$ e $19,94 \%$, na segunda época de amostragem em REC1, REC2, REC3, REC4 e REC5, respectivamente. Isso demonstra que a perturbação da produção agrícola pode levar à redução do C-BMS, que tende a elevar gradualmente à medida que aumenta a resiliência dos ambientes perturbados. A menor discrepância de C-BMS nas REC's em relação à $C N$, na segunda época de amostragem, deve-se ao aumento em $\mathrm{CN}$, que pode estar relacionado com acumulação de resíduos culturais, com as características intrínsecas da cultura e ainda, com as substâncias orgânicas produzidas, especialmente pelas raízes (Belo et al., 2012).

Embora não ter sido observado interação significativa entre os fatores (área e ano de amostragem), pode-se observar que houve redução nos teores de C-BMS na área de MATA entre os anos de amostragem, facto este que pode ser atribuído ao maior gasto de energia para a manutenção da comunidade microbiana, tendo em vista que na segunda época de amostragem houve um aumento acima da média nos índices pluviométricos e de temperatura na região, assim, os microrganismos tenderam a consumir mais substrato para sobreviver ao período de estresse metabólico. Ainda, estudos relatam (Baldrian et al., 2008) uma tendência de aumento da biomassa microbiana durante a fase inicial da sucessão, após esse período os teores de nutrientes geralmente diminuem e a biomassa microbiana pode declinar ou permanecer constante.

A riqueza e abundância de espécies arbóreas implicam uma deposição contínua de substratos orgânicos com composição variada na serapilheira, que favorecem os maiores teores de biomassa microbiana (Souza et al., 2012), observado no solo sob vegetação nativa (Chaer e Tótola, 2007) e nas áreas em restauração ecológica, nas duas épocas de amostragem, quando comparadas ao cultivo de cana-de-açúcar.

Os resultados obtidos quanto aos atributos microbiológicos corroboram com Susyan et al. (2011) que, estudando a sucessão florestal em solos aráveis abandonados na Rússia, encontraram um aumento na biomassa microbiana no solo durante os períodos sucessionais da vegetação, possivelmente devido ao aumento da entrada e acumulação de carbono orgânico no solo.

Quando a biomassa microbiana se encontra sob algum fator limitante (deficiência de um nutriente, acidez, entre outros), a capacidade de utilização do carbono é diminuída (Silva et al., 2007). Anderson e Domsch (1993) afirmaram que o incremento de carbono microbiano, após mudanças no sistema de uso do solo, induz aumento subsequente no carbono orgânico e vice-versa.

Os atributos microbiológicos, observados no presente trabalho, refletem as condições do ambiente para o desenvolvimento dos microrganismos do solo. Áreas com valores mais elevados dos teores de carbono microbiano implicam em maior imobilização temporária de nutrientes devido à maior quantidade de matéria orgânica ativa no solo capaz de manter a decomposição dos resíduos vegetais. Sistemas cultivados geralmente exibem níveis mais baixos de C-BMS do que solos sob vegetação nativa (Kaschuk et al., 2010).

Os teores mais baixos de C-BMS na área de cultivo de cana-de-açúcar podem ser reflexo de impacto e perturbações das formas de manejo sobre a comunidade microbiana decorrentes da adubação nitrogenada, o acúmulo de resíduos decompostos (Frazão et al., 2010) e não se descartam o histórico de queima da cana-de-açúcar para colheita e o revolvimento do solo, fatores associado à forte alteração na estrutura da comunidade edáfica (Rachid et al., 2013), além da quantidade e qualidade dos resíduos vegetais disponíveis à microbiota do solo (Gama-Rodrigues et al., 2008). 
Valores mais elevados da respiração basal (liberação de $\mathrm{C}-\mathrm{CO}_{2}$ ) implicam em maior atividade biológica, que está diretamente relacionada com a disponibilidade de carbono do solo e/ou da biomassa microbiana (Mercante et al., 2006; Allen et al., 2011). Assim, uma alta taxa de respiração pode ser interpretada como uma característica desejável quando se considera que a decomposição dos resíduos orgânicos irá disponibilizar nutrientes para as plantas (Roscoe et al., 2006). Os resultados de $\mathrm{C}-\mathrm{CO}_{2}$ obtidos pela análise de variância indicaram que não houve diferença significativa entre os tratamentos e a época de amostragem.

Ao analisar os valores do quociente metabólico ou da taxa de respiração específica $\left(\mathrm{qCO}_{2}\right)$, que representa a quantidade de $\mathrm{C}-\mathrm{CO}_{2}$ libertada por unidade de biomassa microbiana em determinado tempo (Mercante et al., 2008), verificou-se, pela análise de variância, interação entre as áreas estudadas e a época de amostragem $(p<0,01)$. Em 2012, CN apresentou maior consumo do carbono pela comunidade microbiana, quando comparado às demais áreas avaliadas. $\mathrm{O}$ alto teor de $q \mathrm{CO}_{2}$ observado pode estar relacionado com o sistema de manejo conduzido neste ambiente, que promove rompimento dos macro e microagregados, modificando a estrutura do solo, consequentemente, deixando a matéria orgânica mais suscetível ao ataque microbiano, aumentando a taxa de mineralização e a libertação de $\mathrm{CO}_{2}$ para a atmosfera (Six et al., 2000).

Estas condições podem levar ao desequilíbrio da comunidade microbiana, proporcionando condições de estresse (Anderson e Domsch, 1993; Santos et al., 2015), resultando em maiores perdas de $\mathrm{CO}_{2}$ e menor incorporação de carbono ao tecido celular (Barbosa, 2010).

Na segunda época de amostragem (2014), não houve diferença significativa entre as áreas avaliadas, corroborando com Guimarães et al. (2017), o que pode indicar, que as condições do ambiente estão apropriadas para o desenvolvimento microbiano, as quais podem decorrer da adição de matéria orgânica de boa qualidade ou da eliminação do fator limitante (Chaer e Tótola, 2007). A análise de variância indicou interação significativa $(p<0,01)$ das áreas estudadas e das épocas de amostragem, com média mais elevada em $\mathrm{CN}$ na primeira época de amostragem.
É importante ressaltar que a biomassa microbiana do solo é mais eficiente quanto menor a perda de carbono, como $\mathrm{CO}_{2}$ pela respiração, e uma fração significativa de carbono é incorporada ao tecido microbiano (Gama-Rodrigues, 1999; Roscoe et al., 2006). Um valor alto significa, que a atividade microbiana do solo é baixa na eficiência de conversão de carbono orgânico em carbono microbiano (Wardle e Ghani, 1995). Assim, ecossistemas perturbados pela intensa atividade agrícola, ao serem abandonados, têm como reflexo uma diminuição dos teores de $q \mathrm{CO}_{2}$ ao longo do tempo, indicando que o ecossistema tende para a estabilidade durante os estágios sucessionais.

O quociente microbiano (qMIC) é um índice utilizado para fornecer indicações sobre a qualidade da matéria orgânica dosolo, sendoexpresso pela relação entre $C$ da biomassa microbiana e o carbono orgânico total (Mendes et al., 2011). Ao analisar o resultado da análise de variância obtida neste trabalho, foi observada diferença significativa $(p<0,01)$ entre as épocas de amostragem, indicando aumento no teor de qMIC na segunda época de amostragem.

O aumento do qMIC na segunda época de amostragem, pode estar relacionado com a adição de matéria orgânica de boa qualidade ou com a redução do fator estressante (Chaer e Tótola, 2007) para os microrganismos, como deficiências nutricionais, presença de metais pesados (Wardle, 1994) e revolvimento do solo nas áreas de estudo.

Com a análise de agrupamento hierárquico para o conjunto de atributos microbiológicos (C-BMS, C- $\mathrm{CO}_{2}, \mathrm{qCO}_{2}$ e qMIC) estudados, foi possível a divisão de grupos interpretativos (Figura 1) nos dois períodos de amostragem. Nesta análise, as áreas em restauração ecológica, cultivo de cana-de-açúcar e vegetação nativa foram agrupadas com base no seu grau de semelhança, classificando-as em grupos homogêneos.

$\mathrm{Na}$ interpretação da matriz de similaridade entre as áreas, foi observada a formação de dois grupos interpretáveis (G1 e G2) a partir dos dados dos atributos microbiológicos do solo na camada de 0,00-0,10 m (Figura 1A) no primeiro período de amostragem (2012). Os grupos "G1" e "G2" englobaram a ligação com distância inferior a 100\%, o que permite inferir que a similaridade entre eles 

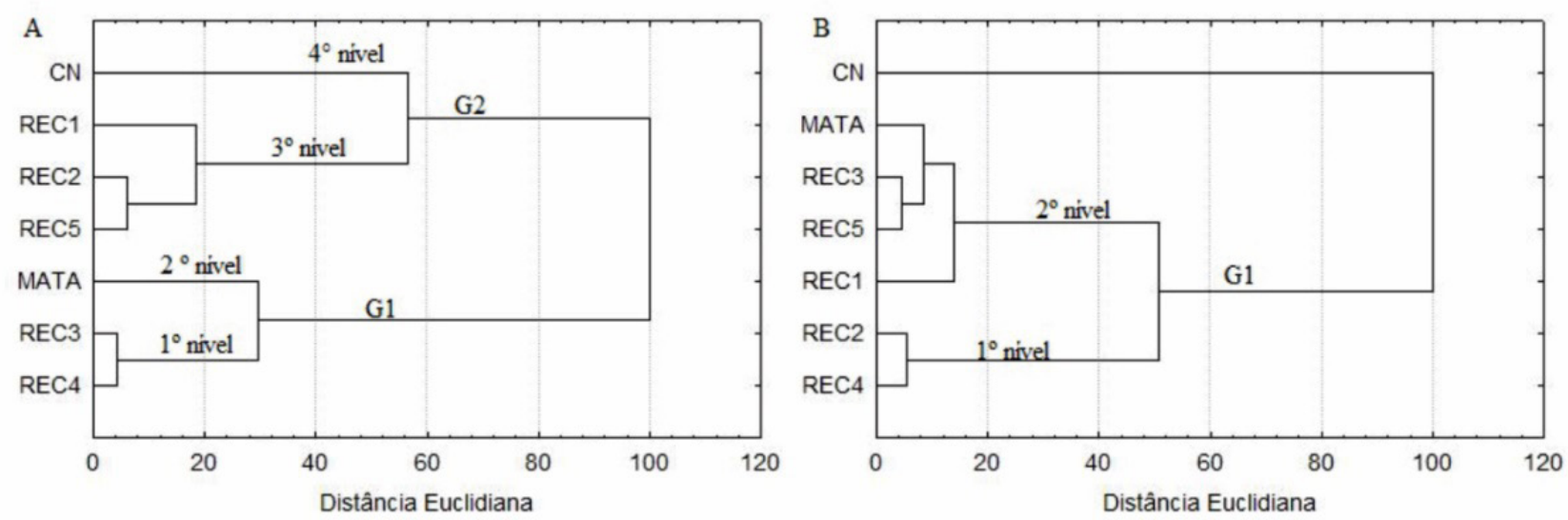

Figura 1 - Dendograma de distância euclidiana dos atributos microbiológicos do solo nas áreas em restauração ecológica, vegetação nativa e cultivo de cana-de-açúcar. (A) época de amostragem 2012, (B) época de amostragem 2014. REC1 (área em restauração ecológica 1), REC2 (área em restauração ecológica 2), REC3 (área em restauração ecológica 3), REC4 (área em restauração ecológica 4), REC5 (área em restauração ecológica 5), CN (Cana-de-açúcar), MATA (fragmento de vegetação nativa de Floresta Estacional Semidecídua).

é nula. Na segunda época de amostragem (2014), houve a formação de apenas um grupo de interpretação, deixando a área de cultivo de cana-de-açúcar separada das demais áreas estudadas (Figura 1B).

A formação do grupo "G1", na primeira época de amostragem, pode ter ocorrido em virtude das áreas em restauração (REC3 e REC4), neste grupamento, apresentarem melhor recuperação dos atributos microbiológicos do solo, quando comparadas às demais áreas em restauração e cultivo de cana-de-açúcar. Estas áreas mostraram mais de 60\% de semelhança com as condições dos remanescentes de vegetação nativa que podem ter sido favorecidos pela manutenção da diversidade de cobertura vegetal e não ocorrência do revolvimento do solo (Portilho et al., 2011).

Em relação à segunda época de amostragem, a formação do grupo "G1" está ligada à evolução do estágio de restauração, que englobou todas as áreas em restauração (REC1 a REC5) com o remanescente de vegetação nativa (MATA), promovendo a separação de tais áreas da área de cultivo de cana-de-açúcar. Este resultado mostra que houve modificação nas estruturas das comunidades de microrganismos do solo e, consequentemente, a manutenção da sua qualidade nas áreas em restauração.
Este resultado é um indicativo que as áreas presentes neste grupo melhoraram a qualidade do solo, refletindo em dissimilaridade com a área sob cultivo de cana-de-açúcar $(\mathrm{CN})$, que pode ser justificado pelo revolvimento do solo no cultivo da cana-de-açúcar que tende a reduzir a manutenção da microbiota, como também, afetar a assimilação de nutrientes pelas plantas (Barbosa, 2010).

Observou-se que, em ambos os períodos de amostragem, pelo menos uma área em restauração ecológica, foi agrupada com a vegetação nativa, fato também encontrado por Yada et al. (2015) ao estudarem os atributos dos solos degradados por mineralização de estanho e em recuperação. Ainda, parece sensato afirmar que para o melhor entendimento dos efeitos das diferentes formas de manejo dos solos na área investigada, além dos atributos microbiológicos dos solos, outros estudos que envolvam a diversidade da microbiota serão importantes para se entender a dinâmica de sucessão em áreas de transição no bioma Cerrado, uma vez que a introdução de espécies vegetais nativas em REC2 não foi o suficiente para influenciar o comportamento microbiológico local, tendo em vista que foi a única área em restauração ecológica a sofrer intervenção humana no processo de restauração. 


\section{CONCLUSÃO}

A restituição de parâmetros de integridade do solo em áreas que sofreram ações de reposição de cobertura vegetal por meio do plantio de mudas nativas ou suspensão do manejo com atividades agrícolas apresentam dinâmica própria, quando comparadas com as áreas testemunhas com relativa proximidade, mesmo tipo e classificação de solo.

Ainda que o tempo de resiliência das áreas seja relativamente curto, o desenvolvimento da cobertura vegetal e o estabelecimento da comunidade microbiana foram importantes para promover a melhoria da qualidade do solo e a restauração de algumas das áreas estudadas.

Mesmo que a área de cultivo de cana-de-açúcar tenha sofrido intensa mecanização e manejo, a mesma apresentou condições microbiológicas favoráveis ao desenvolvimento da cultura e manutenção da atividade biológica no momento da avaliação do experimento.

\section{AGRADECIMENTOS}

EMBRAPA Agropecuária Oeste; FUNDECT, USINA Louis Dreyfus Group Company.

\section{REFERÊNCIAS BIBLIOGRÁFICAS}

Allen, D.E.; Singh, B.P. \& Dalal, R.C. (2011) - Soil health indicators, soil health and climate change: A review of current knowledge. In: Singh, B.P.; Cowie A. \& Chan K. (Eds.) - Soil Health and Climate Change, Soil Biology, vol. 29, p. 25-45. https://doi.org/10.1007/978-3-642-20256-8_2

Anderson, T.H. \& Domsch, K.H. (1990) - Application of eco-physiological quotients $\left(q \mathrm{CO}_{2}\right.$ and $\left.q \mathrm{D}\right)$ on microbial biomasses from soils of different cropping histories. Soil Biology and Biochemistry, vol. 22, n. 2, p. 251-255. https://doi.org/10.1016/0038-0717(90)90094-G

Anderson, T.H. \& Domsch, K.H. (1993) - The metabolic quotient for $\mathrm{CO}_{2}\left(q \mathrm{CO}_{2}\right)$ as a specific activity parameter to assess the effects of environmental conditions, such as $\mathrm{pH}$, on the microbial biomass of forest soils. Soil Biology and Biochemistry, vol. 25, p. 393-395. https://doi.org/10.1016/0038-0717(93)90140-7

Audeh, S.J.S.; Lima, A.C.R.; Cardoso, I.M.; Casalinho, H. \& Jucksch, I.J. (2011) - Qualidade do solo: uma visão etnopedológica em propriedades agrícolas familiares produtoras de fumo orgânico. Revista Brasileira de Agroecologia, vol. 6, n. 3, p. 34-48.

Barbosa, L.A. (2010) - Impacto de sistemas de cultivo orgânico e convencional da cana-de-açúcar nos atributos do solo. Dissertação de Mestrado, Brasília, Universidade de Brasília, Faculdade de Agronomia e Medicina Veterinária, 93 p.

Belo, E.S.; Terra, F.D.; Rotta, L.R.; Vivela, L.A.; Paulino, H.B.; Souza, E.D.; Vilela, L.A.F. \& Carneiro, M.A.C. (2012) - Decomposição de diferentes resíduos orgânicos e efeito na atividade microbiana em um Latossolo Vermelho do Cerrado. Global Science Technology, vol. 5, n. 3, p. 107-116.

Baldrian, P.; Trogl, J.; Frouz, J.; Šnajdr, J.; Valášková, V.; Merhautová, V.; Cajthaml, T. \& Herinková, J. (2008) - Enzyme activities and microbial biomass in topsoil layer during spontaneous succession in spoil heaps after brown coal mining. Soil Biology and Biochemistry, vol. 40, n. 9, p. 2107-2115. https://doi.org/10.1016/j. soilbio.2008.02.019

Chaer, G.M. \& Tótola, M.R. (2007) - Impacto do manejo de resíduos orgânicos durante a reforma de plantios de eucalipto sobre indicadores de qualidade do solo. Revista Brasileira de Ciência do Solo, vol. 31, n. 6, p. 1381-1396. http://dx.doi.org/10.1590/S0100-06832007000600016

Crecchio, C.; Curci, M.; Pellegrino, A.; Ricciuti, P.; Tursi, N. \& Ruggiero, p. (2007) - Soil microbial dynamics and genetic diversity in soil under monoculture wheat grown in different long-term management systems. Soil Biology and Biochemistry, vol. 39, n. 6, p. 1391-1400. https://doi.org/10.1016/j.soilbio.2006.12.016

Dias, L.E.; Franco, A.A. \& Campello, E.F.C. (2007) - Fertilidade do solo e seu manejo em áreas degradadas. In: Novais et al. (Eds.) - Fertilidade do solo. Sociedade brasileira de Ciências do Solo, Viçosa, MG. 1017 p.

Embrapa (2013) - Sistema Brasileiro de Classificação de Solos. 3 ed. Brasília, DF, Empresa Brasileira de Pesquisa Agropecuária, $255 \mathrm{p}$. 
Frazão, L.A.; Piccolo, M.C.; Feigl, B.J.; Cerri, C.C. \& Cerri, C.E.P. (2010) - Inorganic nitrogen, microbial biomass and microbial activity of a sandy Brazilian Cerrado soil under different land uses. Agriculture, Ecosystems \& Environment, vol. 135, n. 3, p. 161-167. https://doi.org/10.1016/j.agee.2009.09.003

Gama-Rodrigues, E.F. (1999) - Biomassa microbiana e ciclagem de nutrientes. In: Santos, G.A. \& Camargo, F.A.O. (Eds.) - Fundamentos da matéria orgânica do solo, Porto Alegre, 654 p.

Gama-Rodrigues, E.F.; Barros, N.F.; Viana, A.P. \& Santos, G.A. (2008) - Alterações na biomassa e na atividade microbiana da serapilheira e do solo, em decorrência da substituição de cobertura florestal nativa por plantações de eucalipto, em diferentes sítios da Região Sudeste do Brasil. Revista Brasileira de Ciência do Solo, vol. 32, n. 4, p. 1489-1499. http://dx.doi.org/10.1590/S0100-06832008000400013

García-Orenes, F.; Morugán-Coronado, A.; Zornoza, R.; Cerdà A \& Scow, K. (2013) - Changes in soil microbial community structure influenced by agricultural management practices in a Mediterranean agro-ecosystem. Plos One, vol. 11, n. 3, art. e0152958. https://doi.org/10.1371/journal.pone.0080522

Guimarães, N.F.; Gallo, A.S.; Fontanetti, A.; Meneghin, S.P.; Souza, M.D.B.; Morinigo, K.P.G. \& Silva, R.F. (2017) - Biomassa e atividade microbiana do solo em diferentes sistemas de cultivo do cafeeiro. Revista de Ciências Agrárias, vol. 40, n. 1, p. 34-44. http://dx.doi.org/10.19084/RCA16041

Hungria, M.; Franchini, J.C.; Brandao-Junior, O.; Kaschuk, G. \& Souza, R.A. (2009) - Soil microbial activity and crop sustainability in a long-term experiment with three soil-tillage and two crop-rotation systems. Applied Soil Ecology, vol. 42, n. 3, p. 288-296. https://doi.org/10.1016/j.apsoil.2009.05.005

Jenkinson, D.S. \& Powlson, D.S. (1976) - The effects of biocidal treatments on metabolism in soil V: A method for measuring soil biomass. Soil Biology and Biochemistry, vol. 8, n. 3, p. 209-213. https:/doi. org/10.1016/0038-0717(76)90005-5

Kaschuk, G.; Alberton, O. \& Hungria, M. (2010) - Three decades of soil microbial biomass studies in Brazilian ecosystems: lessons learned about soil quality and indications for improving sustainability. Soil Biology and Biochemistry, vol. 42, n. 1, p. 1-13. https://doi.org/10.1016/j.soilbio.2009.08.020

Mendes, I.C.; Reis-Junior, F.B.; Hungria, M.; Fernandes, M.F.; Chaer, G.M.; Mercante, F.M. \& Zilli, J.E. (2011) - Microbiologia do solo e sustentabilidade de sistemas agrícolas. In: Faleiro, F.G. \& Andrade, S.R.M. (Eds.) - Biotecnologia: estado da arte e aplicações na agropecuária. Planaltina, DF: Embrapa Cerrados, 730 p.

Mercante, F.M.; Otsuba, A.A.; Silva, R.F.; Hernani, L.C. \& Oliveira, H. (2006) - Monitoramento de parâmetros microbiológicos em área manejadas sob plantio direto na Bacia Hidrográfica do Alto Taquari, MS. Dourados: Embrapa Agropecuária Oeste, boletim informativo 38, 2006. 21 p.

Mercante, F.M.; Silva, R.F.; Francelino, C.S.F.; Cavalheiro, J.C.T. \& Otsubo, A.A. (2008) - Biomassa microbiana, em um Argissolo Vermelho, em diferentes coberturas vegetais, em área cultivada com mandioca. Acta Scientiarum Agronomy, vol. 34, n. 4, p. 479-485. http://dx.doi.org/10.4025/actasciagron.v30i4.5301

Novak, E.; Carvalho, L.A.; Santiago, E.F. \& Portilho, I.I.R. (2017) - Chemical and microbiological attributes under different soil cover. Cerne, vol. 23, n. 1, p. 19-30. http://dx.doi.org/10.1590/01047760201723012228

Portilho, I.I.R.; Crepaldi, R.A.; Borges, C.D.; Silva, R.F.; Salton, J.C. \& Mercante, F.M. (2011) - Fauna invertebrada e atributos físicos e químicos do solo em sistemas de integração lavoura.pecuária. Pesquisa Agropecuária Brasileira, vol. 46, n. 10, p. 1310-1320. http://dx.doi.org/10.1590/S0100-204X2011001000027

Rachid, C.T.C.C.; Santos, A.L.; Piccolo, M.C; Balieiro, F.C.; Coutinho; Peixoto, R.C.; Tiedje, J.M. \& Rosado, A.S. (2013) - Effect of sugarcane burning or Green harvest methods on the Brasilian Cerrado soil bacterial community structure. Plos One, vol. 8, n. 3, art. e59342. https://doi.org/10.1371/journal.pone.0059342

Rangel-Vasconcelos, L.G.T.; Zarin, D.J.; Oliveira, F.A.; Vasconcelos, S.S.; Carvalho, C.J.R. \& Santos, M.M.L.S. (2015) - Effect of water availability on soil microbial biomass in secondary forest in eastern Amazonia. Revista Brasileira de Ciência do Solo, vol. 39, n. 2, p. 377-384. http://dx.doi.org/10.1590/01000683rbcs20140135

Roscoe, R.; Mercante, F.M.; Mendes, I.C.; Reis Junior, F.B.; Santos, J.C.F. \& Hungria, M. (2006) - Biomassa microbiana do solo: Fração mais ativa da matéria orgânica. In: Roscoe, R. (Ed.) - Dinâmica da matéria orgânica do solo em sistemas conservacionistas: Modelagem matemática e métodos auxiliares. Embrapa Agropecuária Oeste, Dourados. 304 p.

Santos, C.A.; Krawulski, C.C.; Bini, D.; Filho, T.G.; Knob, A.; Medina, C.C.; Filho, G.A. \& Nogueira, M.A. (2015) - Reclamation status of a degraded pasture based on soil health indicators. Scientia Agricola, vol. 72, n. 3, p. 195-202. http://dx.doi.org/10.1590/0103-9016-2013-0274 
Silva, M.B.; Kliemann, H.J.; Silveira, P.M. \& Lanna, A.C. (2007) - Atributos biológicos do solo sob influência da cobertura vegetal e do sistema de manejo. Pesquisa Agropecuária Brasileira, vol. 42, n. 12, p. 1755-1761. http://dx.doi.org/10.1590/S0100-204X2007001200013

Singh, B.K.; Tate, K.; Thomas, N.; Ross, D. \& Singh, J. (2011) - Differential effect of afforestation on nitrogenfixing and denitrifying communities and potential implications for nitrogen cycling. Soil Biology and Biochemistry, vol.43, n. 7, p. 1426-1433. https://doi.org/10.1016/j.soilbio.2011.03.007

Six, J.; Paustian, K.; Elliot, E.T. \& Combrink, C. (2000) - Soil structure and organic matter: distribution of aggregate-size classes and aggregate associated carbon. Soil Science Society of America Journal, vol. 64, n. 2, p. 681-689. https://doi.org/10.2136/sssaj2000.642681x

Souza, E.D.; Costa, S.E.V.G.A.; Anghinoni, I.; Lima, C.V.S.; Carvalho, P.C.F. \& Martins, A.P. (2010) Biomassa microbiana do solo em sistema de integração lavoura pecuária em plantio direto, submetido a intensidades de pastejo. Revista Brasileira de Ciência do Solo, vol. 34, n. 1, p. 79-88. http://dx.doi.org/10.1590/ S0100-06832010000100008

Souza, R.A.; Telles, T.S.; Machado, W.; Hungria, M.; Tavares Filho, J. \& Guimarães, M.F. (2012) - Effects of sugarcane harvesting with burning on the chemical and microbiological properties of the soil. Agriculture, Ecosystems \& Environment, vol. 155, p. 1-6. https://doi.org/10.1016/j.agee.2012.03.012

Susyan, E.A.; Wirth, S.; Ananyeva, N.D. \& Stolnikova, E.V. (2011) - Forest succession on abandoned arable soils in European Russia - Impacts on microbial biomass, fungal-bacterial ratio, and basal $\mathrm{CO}_{2}$ respiration activity. European Journal of Soil Biology, vol. 47, n. 3, p. 169-174. https://doi.org/10.1016/j.ejsobi.2011.04.002

Vance, E.D.; Brookes, P.C. \& Jenkinson, D.S. (1987) - An extraction method for measuring soil microbial biomass C. Soil Biology \& Biochemistry, vol. 19, n. 6, p. 703-707. https://doi.org/10.1016/0038-0717(87)90052-6

Wardle, D.A. (1994) - Metodologia para quantificação da biomassa microbiana do solo. In: Hungria, M. \& Araujo, R.S. (Eds.) - Manual de métodos empregados em estudos de microbiologia agrícola. Brasília, DF: EMBRAPA, $542 \mathrm{p}$.

Wardle, D.A. \& Ghani, A. (1995) - A critique of the microbial metabolic quotient $\left(q \mathrm{CO}_{2}\right)$ as a bioindicator of disturbance and ecosystem development. Soil Biology and Biochemistry, vol. 27, n. 12, p. 1601-1610. https:// doi.org/10.1016/0038-0717(95)00093-T

Yada, M.M.; Mingotte, F.L.C.; Melo, W.J.; Melo, G.P.; Melo, V.P.; Longo, R.M. \& Ribeiro, A.I. (2015) - Atributos químicos e bioquímicos em solos degradados por mineração de estanho e em fase de recuperação em ecossistema amazônico. Revista Brasileira de Ciência do Solo, vol. 39, n. 3, p. 714-724. http:// dx.doi.org/10.1590/01000683rbcs20140499 